\title{
Use of Leu M1 and antiepithelial membrane antigen monoclonal antibodies for diagnosing Hodgkin's
} disease

\author{
AS JACK, D CUNNINGHAM,* M SOUKOP,* CHRISTINE N LIDDLE, FD LEE \\ From the Departments of Pathology and *Medical Oncology, Glasgow Royal Infirmary, Scotland
}

SUMMARY Biopsies of 82 patients diagnosed as having Hodgkin's disease were reviewed. Seventeen were reclassified histologically as non-Hodgkin's lymphoma or reactive lymphoid hyperplasia. A substantial number of cases of Hodgkin's disease were negative when stained with Leu M1. Staining for Leu M1 was not found in the cases of non-Hodgkin's lymphoma or reactive lymphoid hyperplasia. With the exception of the lymphocyte predominant nodular subtype of Hodgkin's disease, epithelial membrane antigen staining was seen in a few cases of Hodgkin's disease and nonHodgkin's lymphomas. This was not a useful discriminating feature.

The diagnosis of Hodgkin's disease is clinically important because of the availability of relatively effective treatment. ${ }^{1}$ The established criteria for diagnosis are currently based on the classification of Lukes and Butler. ${ }^{2}$ Recently, the antigranulocyte monoclonal antibody Leu M1 has been reported to react with Reed Sternberg cells in all cases of Hodgkin's disease except the lymphocyte predominant nodular type. ${ }^{34}$ Some cases of lymphocyte predominant nodular Hodgkin's disease have been found to contain Reed Sternberg cells, which are immunoreactive with an antibody to human milk fat globulin antiepithelial membrane antigen (anti-EMA). ${ }^{5}$ Reed Sternberg cells in other subtypes of Hodgkin's disease were negative with this antibody. Positive staining with this antibody has also been reported in some diffuse non-Hodgkin's lymphomas and normal plasma cells. ${ }^{5}$

These findings suggest that reactivity with Leu M1 and anti-EMA, and perhaps other antibodies such as $\mathrm{Ki}-1,{ }^{6}$ may be useful aids in the diagnosis of Hodgkin's disease. To test the usefulness of these antibodies 82 lymph node biopsies that had initially been reported as Hodgkin's disease were reviewed histologically and stained by immunoperoxidase with Leu M1 and anti-EMA.

\section{Material and methods}

TISSUES

Lymph node biopsies from 1969 to 1984 were in-

Accepted for publication 20 November 1985 cluded. These were from patients who had been entered in a therapeutic trial. All the patients were initially diagnosed as having Hodgkin's disease. Tissue was fixed in formol saline with mercuric chloride, formol saline, or neutral buffered formalin, depending on the time of biopsy.

\section{HISTOLOGICAL DIAGNOSIS}

The criteria used were those of Lukes and Butler ${ }^{2}$ with the following modifications: nodular sclerosis was subdivided into NS1 and NS2, as described by Bennett and MacLennan ${ }^{17}$ and cellular phase. ${ }^{89}$ Only one category of lymphocyte depleted Hodgkin's disease was used. For the purposes of this study nonHodgkin's lymphomas were grouped together.

The histological diagnosis was made on reticulin sections stained with haematoxylin and eosin by one of us (ASJ). Problem cases were reviewed jointly with FDL. (About $25 \%$ of cases.) The diagnosis was made without reference to immunohistochemical findings.

\section{IMMUNOHISTOCHEMISTR Y}

Eighty two specimens provided material for study. Sections were dewaxed, and mercuric chloride removed as required. Sections from blocks fixed without mercuric chloride were trypsinised for five to 15 minutes. Endogenous peroxidase was inhibited using acidified methanol containing hydrogen peroxide. After washing in Tris buffered saline at $\mathrm{pH} 7.6$ the sections were incubated in the primary antiserum for 120 minutes. Leu M1 (Becton Dickinson) was used at a dilution of 1/100 and anti-EMA (Dako) at 1/20. 
These dilutions were made in Tris buffer at $\mathrm{pH} 7 \cdot 6$. The sections were washed and antibody binding shown using a rabbit antimouse peroxidase conjugate (Dako) followed by immersion in diaminobenzidine and hydrogen peroxide. The slides were washed, counterstained, and dehydrated.

The antibody concentrations selected were those that gave consistent strong staining of control tissue; a wide range of concentrations having been tested. The tissues used for titration were typical cases of Hodgkin's disease and inflammatory lesions containing granulocytes and plasma cells.

Leu M1 stained slides were assessed. A case was considered to be positive if Reed Sternberg like cells showed membrane or dense dot type cytoplasmic staining (Fig. 1). Borderline cases were those in which one to three cells per section showed weak staining. This included mononuclear cells that did not have specific morphological characteristics of Reed Sternberg cells. Epithelial membrane antigen positivity was assessed the same way. In all the sections stained with Leu M1 densely staining granulocytes were seen. In sections stained with epithelial membrane antigen plasma cells were also stained. These features were ignored in assessment.

\section{Results}

On histological review 12 biopsies were reclassified as non-Hodgkin's lymphoma and five as reactive lymphoid hyperplasia. The remaining 65 cases of Hodg-

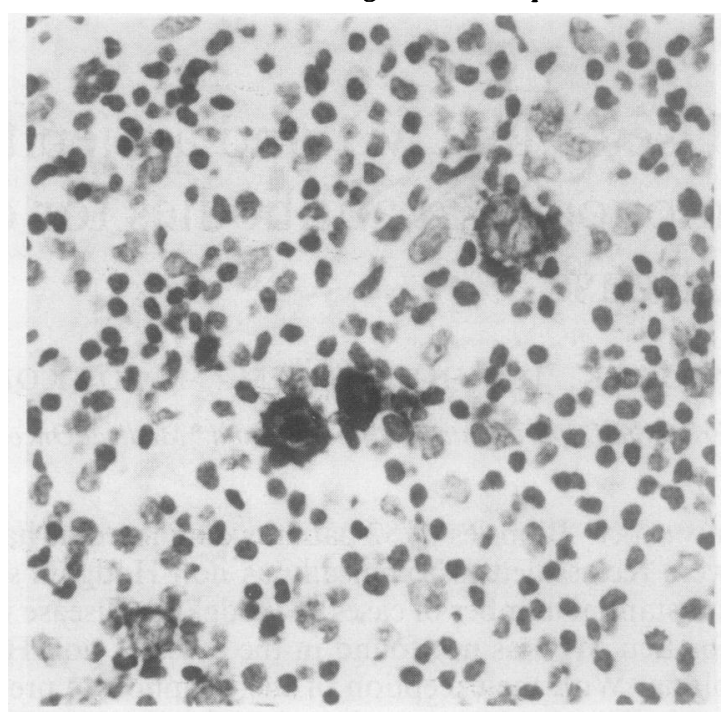

Fig. 1 NS1 Hodgkin's disease showing membrane staining of Reed-Sternberg cells. (Immunoperoxidase. Leu M1.) $\times 502$.

kin's disease were subclassified (Tables 1 and 2). The cases of non-Hodgkin's lymphoma were all diffuse tumours of mixed cell type. No further marker studies could be performed, but on morphological criteria most were thought likely to be peripheral $\mathrm{T}$ cell tumours. One case showed typical features of Lennert's lymphoma. ${ }^{10}$ The case that stained positively with

Table 1

\begin{tabular}{|c|c|c|c|c|}
\hline Histological diagnosis & Leu MI positive ( $\%$ ) & Leu MI borderline ( $\%$ ) & Leu $M 1$ negative ( $\%$ ) & Total \\
\hline $\begin{array}{l}\text { Lymphocyte predominant nodular* } \\
\text { Lymphocyte predominant diffuse } \\
\text { Nodular sclerosis type I } \\
\text { Nodular sclerosis cellular phase } \\
\text { Nodular sclerosis type } 2 \\
\text { Mixed cellularity } \\
\text { Lymphocyte depleted } \\
\text { Non-Hodgkin's lymphoma } \\
\text { Reactive lymphoid tissue }\end{array}$ & $\begin{array}{l}1(14) \\
1(100) \\
13(72) \\
4(44) \\
4(67) \\
16(80) \\
4(100) \\
0(0) \\
0(0)\end{array}$ & $\begin{array}{l}0 \\
0 \\
2(11) \\
1(12) \\
0(0) \\
1(5) \\
0 \\
4(33) \\
0(0)\end{array}$ & $\begin{array}{l}6(86) \\
0 \\
3(17) \\
4(44) \\
2(33) \\
3(15) \\
0 \\
8(67) \\
5(100)\end{array}$ & $\begin{array}{r}7 \\
1 \\
18 \\
9 \\
6 \\
20 \\
4 \\
12 \\
5\end{array}$ \\
\hline
\end{tabular}

*Includes progressive transformation.

Table 2

\begin{tabular}{llllr}
\hline Histological diagnosis & EMA positive (\%) & EMA borderline (\%) & EMA negative (\%) & Total \\
\hline Lymphocyte predominant nodular & $4(57)$ & 0 & $3(43)$ & 7 \\
Lymphocyte predominant diffuse & 0 & 0 & $1(100)$ & 1 \\
Nodular sclerosis type I & 0 & 0 & $18(100)$ & 18 \\
Nodular sclerosis cellular phase & $1(11)$ & 0 & $6(89)$ & 9 \\
Nodular sclerosis type 2 & 0 & 0 & $19(95)$ & 6 \\
Mixed cellularity & $1 *(5)$ & 0 & $4(100)$ & 4 \\
Lymphocyte depleted & $1(9)$ & 0 & $11(91)$ & 12 \\
Non-Hodgkin's lymphoma & 0 & 0 & $5(100)$ & 5 \\
Reactive lymphoid tissue & 0 & & & 7 \\
\hline
\end{tabular}

EMA = epithelial membrane antigen. *Also positive with Leu M1. 


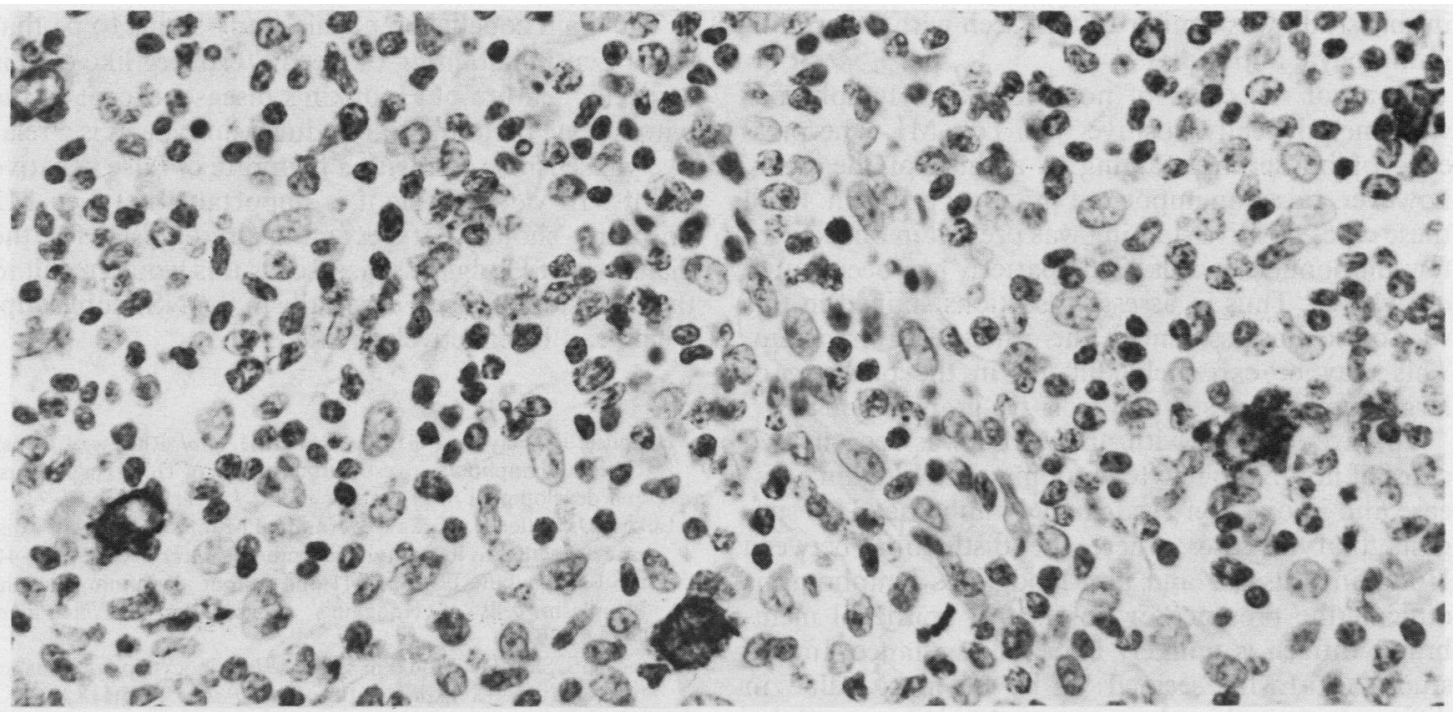

Fig. 2 Case diagnosed as diffuse non-Hodgkin's lymphoma showing cytoplasmic staining with anti-EMA in a few large lymphoid cells. (Immunoperoxidase. Anti-EMA.) × 502.

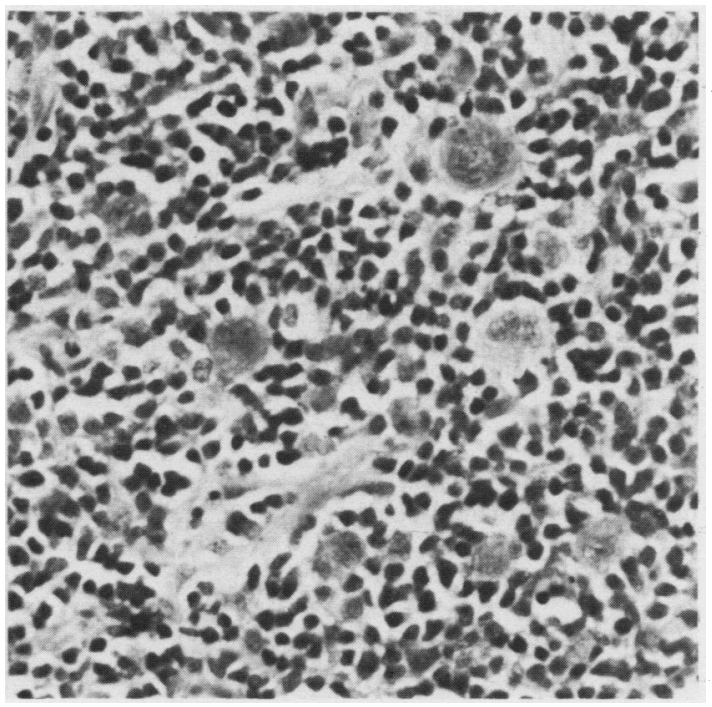

Fig. 3 Histologically typical case of NSI type Hodgkin's disease, in which the Reed-Sternberg cells were unstained with Leu M1. (Haematoxylin and eosin.) x 314 .

anti-EMA contained a population of large lymphoid cells with abundant cytoplasm (Fig. 2).

All the subtypes of Hodgkin's disease, except lymphocyte depleted, showed substantial numbers of cases which were negative, or which showed a borderline staining pattern with Leu M1. This was most commonly seen in the cellular phase of nodular sclerosis. One case of lymphocyte predominant nod- ular Hodgkin's disease showed strong positive staining with Leu M1 in multilobated Reed Sternberg cells. None of the cases of non-Hodgkin's lymphomas showed definite positive staining, although one third were classified as borderline (Table 1).

Only seven cases showed staining with anti-EMA, including the four cases of lymphocyte predominant nodular Hodgkin's disease. One case of mixed cellularity Hodgkin's disease was positive with both epithelial membrane antigen and Leu M1 (Table 2).

There was no apparent correlation between fixative used and negative reaction with Leu M1 and antiEMA. In addition, positively staining granulocytes and plasma cells provided an internal positive control in every section.

\section{Discussion}

Many biopsies in this study presented difficult diagnostic problems; using strict criteria 65 were eventually classified as Hodgkin's disease. In contrast to other studies $^{356}$ there was not a perfect correlation between histological diagnosis and Leu M1 immunoreactivity. Excluding the lymphocyte predominant nodular subtype of Hodgkin's disease, $22 \%$ of cases of Hodgkin's disease were either negative or showed borderline staining with this antibody. In the case of the nodular sclerosis group $40 \%$ were negative. These observations were consistent with the finding that in every case of Hodgkin's disease a variable number of Reed-Sternberg cells were unstained. The proportion of cases of Hodgkin's disease that reacted to Leu M1 
in this study was similar to that seen with other antigranulocyte antibodies. ${ }^{11}$

None of the cases of non-Hodgkin's lymphomas was unequivocally positive with Leu M1. The presence of borderline staining in a third of the cases, however, raises an important practical problem. In all these cases positive staining was present in only a very few mononuclear cells. This pattern has been noted elsewhere. ${ }^{3}$ Thus in assessing sections stained in this way critical assessment of the cell type is important. This may be extremely difficult in the presence of large numbers of strongly positive granulocytes.

Positive staining with anti-EMA was seen in two cases of non-lymphocyte predominant Hodgkin's disease and in one non-Hodgkin's lymphoma. This suggests that in cases where the distinction between Hodgkin's disease and non-Hodgkin's lymphoma is difficult the presence or absence of epithelial membrane antigen is unlikely to be of assistance. In this study anti-EMA seemed to be of most value in differentiating lymphocyte predominant nodular Hodgkin's disease from reactive hyperplasia.

Studies of this type are critically dependent on correct histological diagnosis. In the case of Hodgkin's disease this may prove difficult, as is illustrated by the number of cases in this study that were reclassified, after being originally diagnosed as Hodgkin's disease. One factor responsible for this seemingly high proportion of cases is that during the period covered by the study (1969-84) the histological criteria currently in use were only gradually gaining acceptance. This raises the question as to whether the histological diagnosis was accurate in the cases that were found to be negative with Leu M1. This argument would be tenable if negativity with Leu M1 correlated with difficulty in histological diagnosis. This was the case in some of the biopsies classified as mixed cellularity or NS2, but other cases were histologically typical (Fig. 3).
The main conclusion of this study seems to be that while Leu M1 staining of Reed Sternberg like cells is a specific feature of Hodgkin's disease, except in the lymphocyte predominant nodular subtype, it is a relatively insensitive test with a high rate of false negative results. In view of this it is important that Leu M1 positivity should not become a prerequisite for the diagnosis of Hodgkin's disease, as this would result in the exclusion of cases, especially in the NS1 subgroup, which can be effectively treated.

\section{References}

${ }^{1}$ Haybittle JL, Hayhoe FGJ, Easterling MJ, et al. Review of British National lymphoma Investigation Studies of Hodgkin's disease and development of prognostic index. Lancet 1985;i:967-73.

${ }^{2}$ Lukes RJ, Butler JJ, Hicks EB. National history of Hodgkin's disease as related to its pathologic picture. Cancer 1966;19:317-44. $\omega$

${ }^{3}$ Hsu Su-Ming, Jaffe ES. Leu M1 and peanut agglutinin stain the Neoplasm cells of Hodgkin's disease. Am J Clin Pathol 1984;82:29-32.

${ }^{4}$ Pinkus GS, Said JW. Hodgkin's disease lymphocyte predominance type, nodular-a distinct entity. Am J Pathol 1985;118:1-6.

${ }^{5}$ Delsol G, Gatter KC, Stein H, et al. Human lymphoid cells express epithelial membrane antigen. Lancet 1984;ii:1124-9.

${ }^{6}$ Stein H, Lennert K, Feller AC, Mason DY. Immunohistological analysis of human lymphoma: correlation of histological and immunological categories. Adv in Cancer Res 1984;42:67-147.

${ }^{7}$ Bennett MH, MacLennan KA, Easterling MJ, et al. The prognostic significance of cellular subtypes in nodular sclerosing Hodgkin's disease: an analysis of 271 non-laparotomied cases (BNL1 Report 22). Clin Radiol 1983;34:497-501.

${ }^{8}$ Lukes RJ. Criteria for involvement of lymph node, bone marrow, spleen and liver in Hodgkin's disease. Cancer Res 1971;31:1755-67.

${ }^{9}$ Colby TV, Hoppe RT, Warnke RA. Hodgkin's disease: a clinicopathologic study of 659 cases. Cancer 1981;49:1848-58.

${ }^{10}$ Burke JS, Butler JJ. Lennert's lymphoma. Lancet 1976;ii:1369-71.

${ }^{11}$ Stein $\mathbf{H}$, Uchanska-Ziegler B, Gerdes J, Ziegler A, Wernet P. Hodgkin and Sternberg-Reed cells contain antigens specific to late cells of granulpoiesis. Int J Cancer 1982;29:283-90.

Requests for reprints to: Dr AS Jack, Department of Pathology, Royal Infirmary, Glasgow G4 OST, Scotland. 